\title{
Multiple Primary Cancer and Survival After Lumpectomy with or without Radiotherapy for Ductal Carcinoma in Situ: A Propensity-Score- Matching Study
}

\author{
Yitong Li \\ Second Xiangya Hospital of Central South University \\ Dengjie Ouyang \\ Second Xiangya Hospital of Central South University \\ Qitong Chen \\ Second Xiangya Hospital of Central South University \\ Tao Hong \\ Second Xiangya Hospital of Central South University \\ Wenjun Yi ( $\nabla$ yiwenjun@csu.edu.cn ) \\ Second Xiangya Hospital of Central South University
}

\section{Research Article}

Keywords: MPC, DCIS, radiotherapy

Posted Date: September 21st, 2021

DOl: https://doi.org/10.21203/rs.3.rs-857840/v1

License: (c) (i) This work is licensed under a Creative Commons Attribution 4.0 International License.

Read Full License 
Multiple primary cancer and survival after lumpectomy with or without radiotherapy for ductal carcinoma in situ: A propensityscore-matching study

\section{Authors}

Yitong $\mathrm{Li}^{1}$, Dengjie Ouyang ${ }^{1, \#}$, Qitong Chen ${ }^{1, \#}$, Tao Hong ${ }^{1, \#}$, Wenjun $\mathrm{Yi}^{1,{ }^{1}}$ \#equal contributors

Affiliation(s) list:

1. Department of General Surgery, The Second Xiangya Hospital, Central South University, Changsha, 410000, Hunan, China;

Corresponding Authors list:

Prof Wenjun Yi, yiwenjun@csu.edu.cn. 


\begin{abstract}
Background: Radiotherapy combined with breast-conserving surgery is widely performed in patients with ductal carcinoma in situ (DCIS). This research was conducted to evaluate the use of radiotherapy to reduce the risk of multiple primary cancer (MPC) and mortality in DCIS patients.

Methods: 108,416 patients first diagnosed with DCIS between 1998 and 2015 who received lumpectomy in the Surveillance, Epidemiology, and End Results (SEER) 18 database were included. Age, race, year of diagnosis, laterality, pathologic grade, surgery, radiation, estrogen receptor status, progesterone receptor status, tumor size and vital status were extracted. A comparison of lumpectomy vs. lumpectomy plus radiotherapy was performed using 1:1 propensity score-based matching.
\end{abstract}

Results: Of the 108,416 patients, 39,039 patients were treated with lumpectomy alone, and 69,377 were treated with lumpectomy and radiotherapy. The adjusted hazard ratio (HR) for death was 0.801 and 0.7444 for occurrence of MPC in the lumpectomy and radiotherapy vs. lumpectomy alone groups, respectively.

Conclusion: Lumpectomy plus radiotherapy is associated with a significant reduction in mortality and risk of MPC, mainly second primary BC. Younger patients, Black women with high-grade tumors were likely to benefit most. Radiotherapy reduced the risk of occurrence rather than the mortality of MPC patients to reduce the overall mortality.

Key words: MPC, DCIS, radiotherapy 


\section{Introduction}

Ductal carcinoma in situ (DCIS) is a noninvasive condition in which abnormal cells are found in the lining of a breast duct ${ }^{1}$. Since the 1970s, multicenter prospective randomized controlled studies on breast-conserving surgery for early breast cancer (BC) patients have shown no significant difference in disease-free and overall survival in such patients compared to those receiving total mastectomy ${ }^{2,3}$. In addition, radiotherapy plays an essential role in breast-conserving surgery ${ }^{4,5}$, resulting in low mortality for patients with DCIS ${ }^{6,7}$. While long-term survivors benefit from systemic treatment, the frequency of multiple primary cancer (MPC) diagnosis after BC has been increasing, with MPC becoming one of the leading causes of death ${ }^{8}$.

Multiple primary cancer (MPC) is defined as more than 2 separate original tumors that simultaneously or successively occur in the same or different organs ${ }^{9}$. Previous studies reported a greater risk of MPC among patients with an initial diagnosis of breast cancer at the age of $<50$ years and in Black women with an ER-/PR- status than in Other patients ${ }^{10}$. A retrospective study did not demonstrate an association between systemic therapy and increased risks of MPC'11, while a nested case-control study using the SEER database suggested that radiotherapy $(\mathrm{HR}=1.33)$ was related to the risk of second primary malignancy after $\mathrm{BC}^{12}$.

To further study the risk of MPC and mortality in DCIS patients, we conducted a population-based study of women with DCIS using the Surveillance, Epidemiology, and End Results (SEER) database. We extracted data on age and year of diagnosis, laterality, pathologic grade, treatment (surgery and radiation), estrogen receptor (ER)status, progesterone receptor (PR) status, tumor size, MPC and death from BC. We sought to determine whether radiotherapy was associated with a reduced risk of BC death and MPC in this cohort of women and to identify subgroups of women who might benefit the most from radiotherapy.

\section{Materials and methods}

\section{Study population}

SEER 18 Regs Custom Data (with additional treatment fields), Nov 2017 Sub (1973-2015 varying) was used to identify the cohort of women for this study. Since estrogen receptor status and progesterone receptor status were provided after 1998, we selected all female patients with confirmed stage $0 \mathrm{BC}$ coded by the $6^{\text {th }}$ edition breast-adjusted AJCC guidelines between 1998-2015 ${ }^{13}$. Among the cases classified as Tis, we excluded those associated with lobular carcinoma in situ, Paget's disease, and an unknown or invasive histology type. We excluded cases with unknown laterality and unknown or no surgical intervention for the primary tumor. We also excluded patients who underwent mastectomy to study those who underwent lumpectomy only. The flow chart of selection is provided in Figure 1.The research protocol was approved by the research ethics board of the Second Xiangya Hospital, Central South University, China. All methods were carried out in accordance with relevant guidelines and regulations. Since this is a retrospective study, the need for the informed consent was waived by The Second Xiangya Hospital. However, we submitted a data use agreement form to access the SEER Research Data File. Upon acceptance of the agreement, the SEER*Stat software and data files were downloaded directly from the SEER website. 


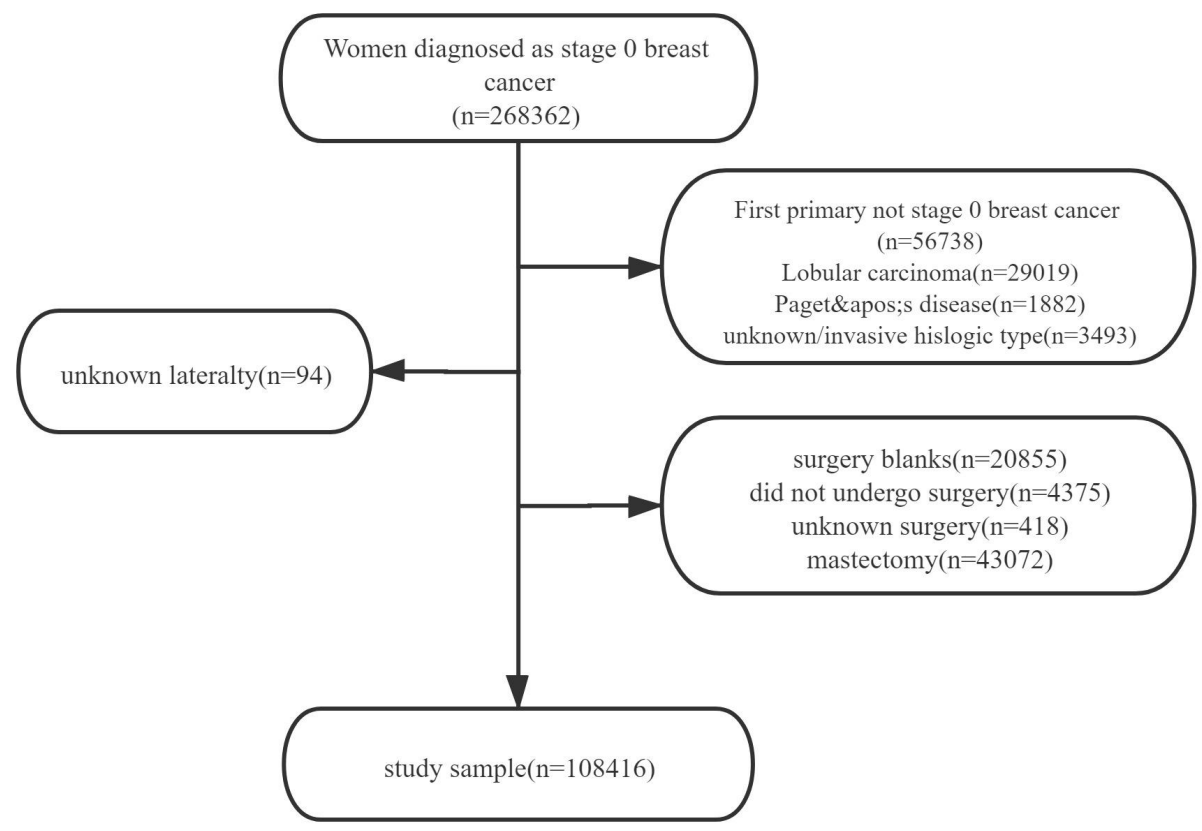

Figure 1. Flow chart for the selection of study samples

\section{Variables}

The SEER data included patient age at diagnosis, race (White, Black, or Other (American Indian/AK Native or Asian/Pacific Islander)), year of diagnosis, laterality, pathologic grade, surgery of the primary site, radiation, estrogen receptor status, progesterone receptor status, tumor size and vital status, which are associated with patients' outcome. We determined 2 time intervals: the time from DCIS to death and the time between the diagnosis of $\mathrm{BC}$ and a second primary cancer incidence (contra-lateral breast cancer and ipsilateral breast cancer included). We divided all the patients diagnosed with a second primary $\mathrm{BC}$ after DCIS into ipsilateral and contralateral groups to compare the laterality of the two primary cancers. Patients eligible for study were categorized into 2 groups: lumpectomy without radiation and lumpectomy with radiation. The groups were compared for the variables above, and differences were evaluated using standardized differences.

\section{Statistical Analysis}

To analyze the association between OS and treatment modality, we performed a propensity score-based model. We calculated the propensity score using logit first an then patient were 1:1 matched for age at diagnosis, race (White, Black, or Other (American Indian/AK Native or Asian/Pacific Islander)), year of diagnosis, laterality, pathologic grade, estrogen receptor status, progesterone receptor status and tumor size based on their propensity score. Caliper matching was performed by matching participants who were within 0.2 times the standard deviation of their propensity score ${ }^{14}$. A standardized difference of greater than 0.1 was considered a meaningful imbalance between comparison groups ${ }^{15}$. P value was also shown after chi square test.

To determine the extent to which radiation was associated with decreased risk of death and MPC in these various subgroups, we compared the hazard ratios (HRs) in different treatment brought to the subgroups, and the results were shown in the forest plots .

The incidence of second primary cancer was compared with the expected incidence in the general population by calculating standardized incidence ratios (SIRs) and 95\% confidence intervals (CIs). In this case, SIR in the general population is 1, and the incidence risk increases when $\operatorname{SIR}>1$, decreases when $\operatorname{SIR}<1$ respectively. SIRs were calculated overall and stratified by clinical parameters. The significance between SIR was analyzed using iri in Stata 14.

Cumulative survival curves after DCIS for each patient variable were constructed using the Kaplan-Meier method and were analyzed using the log-rank test in GraphPad Prism 7. To analyze both mortality and MPC and avoid the biases caused by 
competing events censored, we looked into competing risk model. Cumulative incidence function for competing risks data has been proposed and Fine and Gray test valued the difference using 'cmprsk' package. We also conducted Kaplan-Meier curves stratified by MPC and only one primary cancer group to observe the mortality difference. Age at diagnosis, race (White, Black, or Other (American Indian/AK Native or Asian/Pacific Islander)),pathologic grade, estrogen receptor status, progesterone receptor status and patients' prognosis were identified by Cox proportional hazards regression, and the associated hazard ratios (HRs) were reported. Statistical analysis was performed using R version 3.6.3 software (http://www.r-project.org/).

\section{Results}

\section{Baseline clinical characteristics}

Among the 108,416 patients involved in the cohort, 39,039 patients were treated with lumpectomy alone, and 69,377 were treated with lumpectomy and radiotherapy (Table 1). A greater proportion of patients receiving lumpectomy alone were aged +70 years versus lumpectomy plus radiation therapy. Slightly more Black patients and patients of Other races (American Indian/AK Native or Asian/Pacific Islander) received lumpectomy than radiotherapy plus lumpectomy (Black: 7,502 [10.8\%] vs. 3,958 [10.1\%]; Other races: 7,317 [10.5\%] vs. 3,823 [9.8\%]). The use of radiotherapy also increased with increasing tumor grade. Radiotherapy was more commonly used when the estrogen receptor status or the progesterone receptor status was positive.

Table 1. Baseline characteristics of patients with ductal carcinoma in situ

Table 1. Baseline characteristics of patients with ductal carcinoma in situ

\begin{tabular}{|c|c|c|c|c|}
\hline \multirow{3}{*}{$\frac{\text { Value }}{\text { Patients }}$} & \multicolumn{3}{|c|}{$\mathbf{N}(\%)$} & \multirow{3}{*}{$\mathbf{P}$} \\
\hline & \multicolumn{3}{|c|}{ Lumpectomy alone Lumpectomy plus radiotherapy SMD } & \\
\hline & 39,039 & 69,377 & & \\
\hline \multicolumn{5}{|l|}{ Age at diagnosis, $y$} \\
\hline$<40$ & $864(2.2)$ & $1,440(2.1)$ & & \\
\hline $40-54$ & $11,943(30.6)$ & $25,388(36.6)$ & \multirow{2}{*}{\multicolumn{2}{|c|}{$0.303<0.001$}} \\
\hline $55-69$ & $14,305(36.6)$ & $30,198(43.5)$ & & \\
\hline $70+$ & $11,927(30.6)$ & $12,351(17.8)$ & & \\
\hline \multicolumn{5}{|l|}{ Race } \\
\hline Black & $3,958(10.1)$ & $7,502(10.8)$ & & \\
\hline Other & $3,823(9.8)$ & $7,317(10.5)$ & \multirow{2}{*}{\multicolumn{2}{|c|}{$0.093<0.001$}} \\
\hline Unknown & $520(1.3)$ & $343(0.5)$ & & \\
\hline White & $30,738(78.7)$ & $54,215(78.1)$ & & \\
\hline \multicolumn{5}{|l|}{ Year of diagnosis } \\
\hline $1998-2004$ & $13,621(34.9)$ & $19,920(28.7)$ & & \\
\hline 2005-2009 & $11,176(28.6)$ & $21,669(31.2)$ & \multicolumn{2}{|c|}{$0.133<0.001$} \\
\hline $2010-2015$ & $14,242(36.5)$ & $27,788(40.1)$ & & \\
\hline Laterality $=$ right & $19,033(48.8)$ & $33,932(48.9)$ & 0.003 & 0.627 \\
\hline \multicolumn{5}{|l|}{ Grade } \\
\hline I & $6,843(17.5)$ & $7,569(10.9)$ & & \\
\hline II & $15,056(38.6)$ & $24,538(35.4)$ & \multirow{2}{*}{\multicolumn{2}{|c|}{$0.35<0.001$}} \\
\hline III & $10,063(25.8)$ & $28,295(40.8)$ & & \\
\hline Unknown & $7,077(18.1)$ & 8,975 (12.9) & & \\
\hline \multicolumn{5}{|c|}{ Estrogen receptor status } \\
\hline Negative & $2,329(6.0)$ & $7,128(10.3)$ & \multirow{2}{*}{\multicolumn{2}{|c|}{$0.314<0.001$}} \\
\hline Positive & $20,459(52.4)$ & $43,114(62.1)$ & & \\
\hline
\end{tabular}



Unknown
$16,251(41.6)$
$19,135(27.6)$

Progesterone receptor status

\begin{tabular}{lccc} 
Negative & $3,914(10.0)$ & $11,372(16.4)$ & \\
Positive & $17,244(44.2)$ & $35,899(51.7)$ & $0.308<0.001$ \\
Unknown & $17,881(45.8)$ & $22,106(31.9)$ & \\
umor size, cm & & & \\
$<2.0$ & $22,195(56.9)$ & $41,597(60.0)$ & \\
$2.0-4.9$ & $4,481(11.5)$ & $9,951(14.3)$ & $0.143<0.001$ \\
$\geq 5.0$ & $1,075(2.8)$ & $1,572(2.3)$ & \\
Unknown & $11,288(28.9)$ & $16,257(23.4)$ & \\
\hline
\end{tabular}

Abbreviations: SMD, standardized mean difference

\section{Radiotherapy reduced the risk of mortality and MPC}

In the matched comparison of patients treated with lumpectomy and radiotherapy vs,lumpectomy, radiotherapy was associated with a reduced risk of death (HR=0.7444, 95\%CI: 0.7139-0.7761, $p<0.0001$ ) (Fig. 2a). In addition, we determined the time interval between the first diagnosis of DCIS and the occurrence of multiple primary cancer (MPC) among the 36,688 propensity-matched pairs. The adjusted HR for MPC in the lumpectomy and radiotherapy vs. lumpectomy alone groups was 0.801 (95\%CI: 0.7728-0.8301) (Fig. 2b). Moreover, radiotherapy benefited disease-specific specific survival.(Fig. s1) As radiation reduces MPC but may increase MPC free mortality, competing risk analysis were used to look into. We compared breast cancer specific mortality competing death due to other causes (Fig.s2a) and MPC with competing risk of death without MPC (Fig.s2b). Lumpectomy plus radiotherapy reduced the cumulative incidence of breast cancer specific death and other tumor specific death. Meanwhile, radiotherapy benefited on reducing the risk of MPC, and MPC free mortality.

Figure 2. Kaplan-Meier curves of the cumulative hazard of death (A) and the cumulative hazard of multiple primary cancer (B) for DCIS patients (all $\mathrm{p}<0.0001$, log-rank test)

\section{Younger Black women and high-grade tumors benefited the most from}

\section{radiotherapy}

A
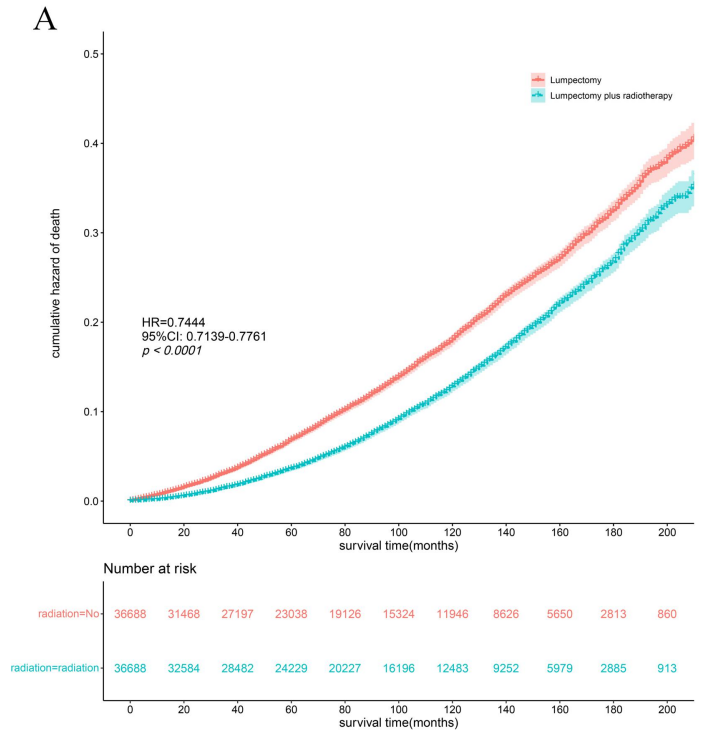

B
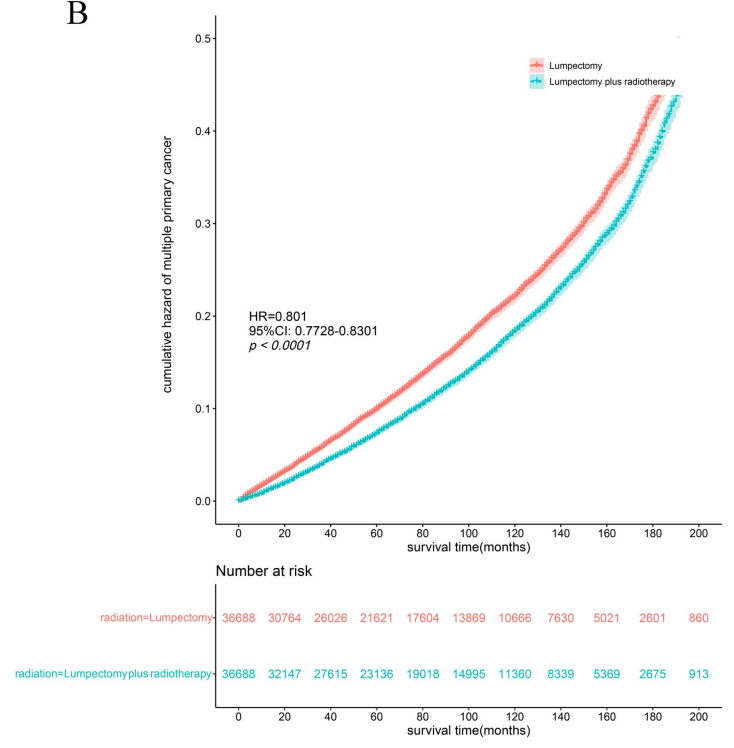

Next, we assessed the protective effect of radiotherapy on mortality and MPC incidence in different subgroups based on the PSM model (Fig. 3). All subgroups could benefit from radiotherapy, but the degree of benefit varied among different subgroups. 
For the risk of MPC, younger patients could benefit more than the elder patients. Black women benefited more than White women $(0.778$ vs. 0.832$)$. The HR was 0.894 for patients with grade I tumors, 0.731 for patients with grade II tumors, and 0.683 for patients with high-grade tumors (grade III). The subgroup containing Black women younger than 40 years with high-grade tumors benefited more than other subgroups in terms of mortality (Fig. 3a). ER negative patients could benefit more compared with those ER+ patients.

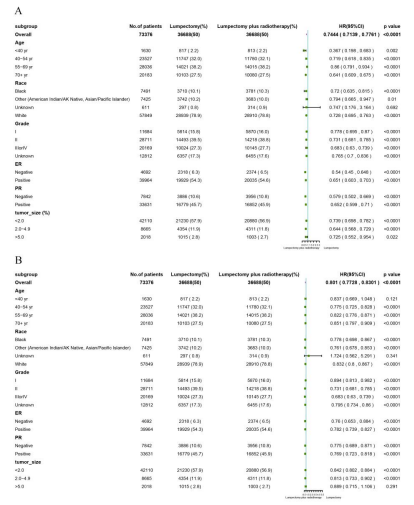

Figure 3. Hazard ratios for mortality (A) and the risk of multiple primary cancer (B) in various subgroups

\section{Effect of radiotherapy on the standardized incidence ratio differed by tumor type}

Next, we calculated and compared the standardized incidence ratios (SIRs) among DCIS patients treated with lumpectomy vs. lumpectomy plus radiotherapy (Fig. 4). The rates of MPC that occurred at all sites among those treated with radiation (lumpectomy plus radiotherapy $\mathrm{SIR}=1.34,95 \% \mathrm{CI}$ : 1.32-1.39; lumpectomy alone $\mathrm{SIR}=1.36,95 \% \mathrm{CI}$ : 1.34-1.38) were lower than those among patients treated with other therapies, while both SIRs were higher than those for the general female population in the SEER database. The SIRs varied across different types of second primary cancer. For second BC, which accounted for the largest proportion of second primary cancer, the SIR was 2.11 (95\%CI: 2.06-2.17) for patients treated with radiation and 2.19 (95\%CI: 2.13-2.24) for patients treated without radiation. Besides, the protective effect was only significant in second female breast cancer $(\mathrm{p}=0.0454)$ and there were no stastical significances in non-breast cancer..

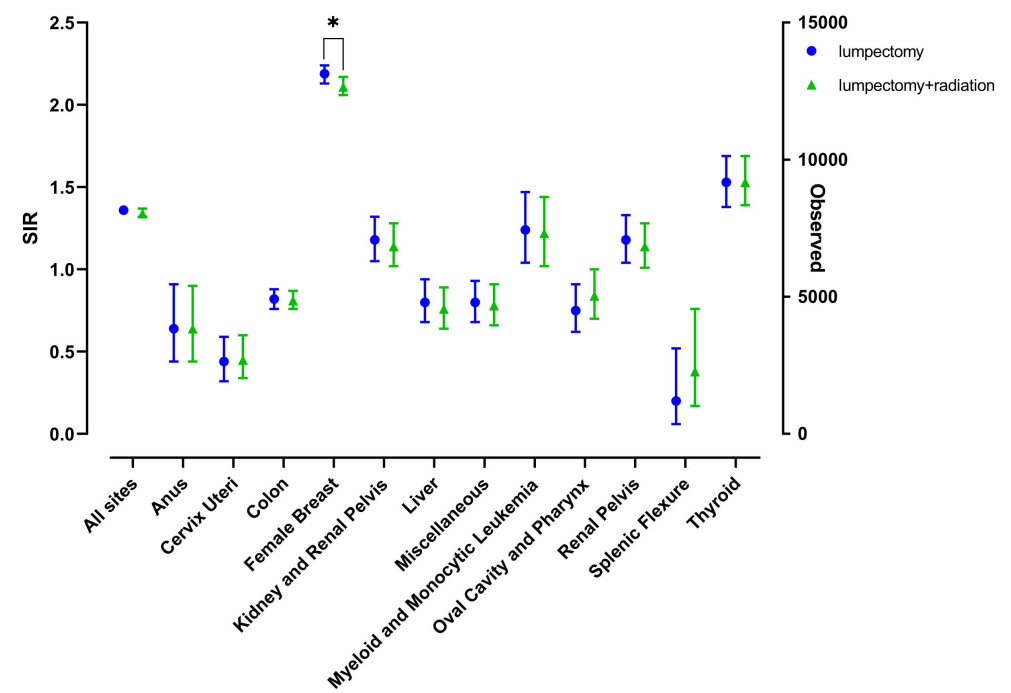

Figure 4. Standardized incidence ratios (SIRs) of second primary cancer after DCIS for patients treated with lumpectomy or lumpectomy plus radiation coded by "site recode B ICD-O-3/WHO 2008" showing a significant 
difference $(\mathrm{p}<0.05)$ compared with those for the general population ( * indicated $p<0.05$ between lumpectomy and lumpectomy+radiation)

\section{Radiotherapy reduced both contralateral and ipsilateral second breast cancer incidence}

The incidence rate of second breast cancer was reduced by $1.83 \%$ with radiotherapy (from $5.93 \%(2,177 / 36,688)$ to $4.10 \%$ $(1,504 / 36,688))$ in the matched cohort. Radiotherapy did not reduce the incidence rate of contralateral second primary breast cancer incidence rate significantly (from $2.45 \%(901 / 36,688)$ to $2.39 \%(879 / 36,688)$ ). The $\%$ reduction was greater when the second breast cancer occurred ipsilaterally (from $3.48 \%(1,276 / 36,688)$ to $1.7 \%(625 / 36,688)$ ).

Radiotherapy reduced the risk of occurrence of MPC rather than the mortality of MPC patients to reduce the overall mortality

To elucidate the association between radiotherapy and the prognosis of patients with only one primary cancer, KM curve analyses were performed. MPC patients had a higher risk of death than patients with only one primary cancer, and the HR was 1.944 (95\%CI: 0.848-2.045) (Fig. 5a). Radiotherapy reduced the risk of death in only the group of patients with one primary cancer (Fig. 5c), and no statistically significant reduction in the risk of death was found in the MPC group between patients treated with lumpectomy alone vs. those treated with lumpectomy plus radiotherapy (Fig. 5b).
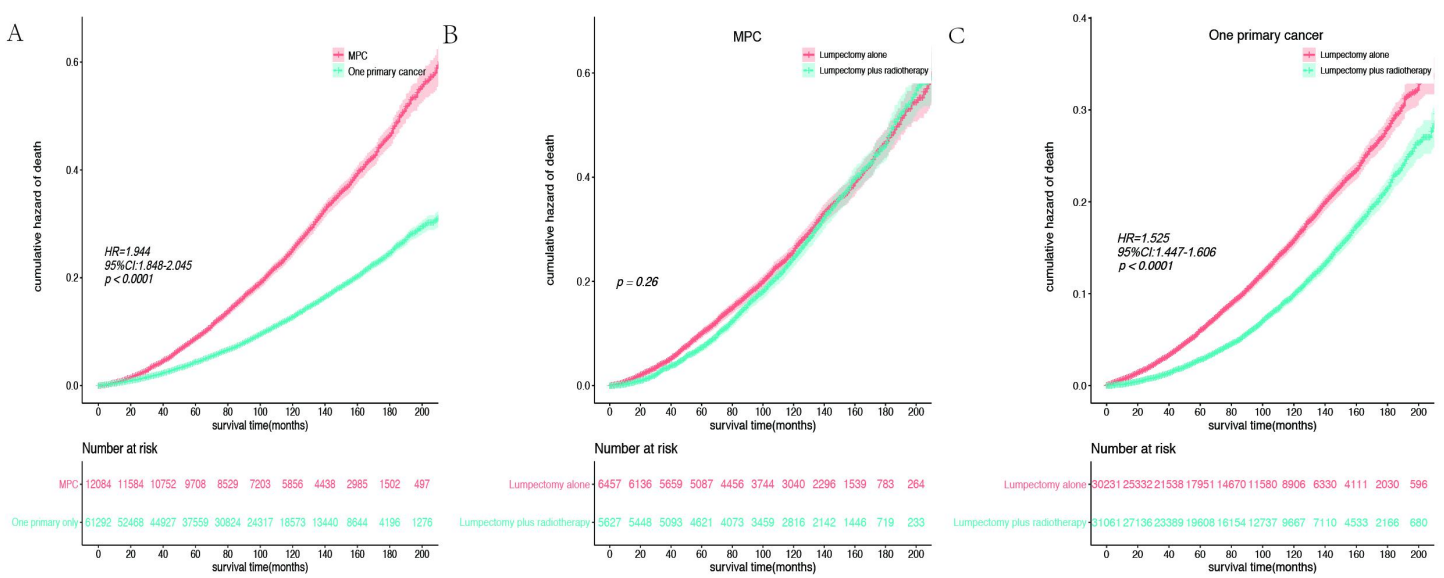

Figure 5. KM curves of patients overall (A); in the MPC group (B) and in the only one primary cancer group (C).

\section{Discussion}

Our study is the first to characterize the risk of MPC and death in DCIS patients in a large population using propensity score matching analysis. Among those with DCIS treated with lumpectomy, the majority of patients received radiotherapy, and these patients were middle-aged and elderly and were more likely to have high-grade disease, ER-positive status, PR-positive status and smaller tumor size than those who did not undergo radiation (Table 1). These factors were accounted for in the matched analysis, and propensity scoring was used to balance the baseline characteristics between the groups (Table s1). Besides, competing risk model minimalized the biases when competing events were censored. Therefore, we believe that the reductions in the risk of death and MPC are a result of the radiotherapy.

In the present study, the incidence of a second primary breast, kidney, renal pelvis and thyroid cancer as well as leukemia after DCIS increased, similar to previous studies ${ }^{16,17}$, possibly related to the mutated driven gene and endocrine therapy, specifically tamoxifen, had a slightly increased risk of uterine primary. As previously established, this increased risk of MPC is partially attributable to genetic and environmental influences. Importantly, we found that radiotherapy could reduce the risk of MPC by $20 \%$, and several reports support our conclusion. Kaire Innos et al ${ }^{18}$ concluded that the use of radiation therapy appears to be protective against the occurrence of subsequent ipsilateral invasive cancer after studying California Cancer Registry data on 23,547 women with DCIS first diagnosed in 1988-1999. Carolyn J. Kushner et al ${ }^{19}$ studied a dataset comprising 755 women with DCIS $(n=135)$ or stage I-II breast carcinoma $(n=620)$ and found that high-dose radiotherapy ( $>63$ Grays) was not associated with a significantly increased risk of SMN. William Beaumont Hospital ${ }^{20}$ reported that the overall 10-year and 
15-year rates of second malignancy among the surgery and radiotherapy (S+RT) and surgery alone cohorts were $14.2 \%$ and $24.2 \%$, and $16.4 \%$ and $22.6 \%$, respectively $(\mathrm{p}=0.668)$. The 15 -year second contralateral BC rate was $14.2 \%$ in the $\mathrm{S}+\mathrm{RT}$ cohort and $10.3 \%$ in the surgery alone cohort $(\mathrm{p}=0.439)$. A newest observational cohort study from the NHS Breast Screening Programme and the National Cancer Registration and Analysis Service observed patients with breast-conserving surgery (BCS) + radiotherapy had lower risk of invasive breast cancer after DCIS compared to those performed $\mathrm{BCS}^{21}$. There are several possible underlying mechanisms: 1) There is statistical evidence of departure from a linear dose-response, with a downward curvature seen at high doses, likely resulting from radiation-induced cell sterilization ${ }^{22}$. 2) High-dose radiation may suppress estrogen production by relevant target cells, as well as androgen production by other cells (with androgen having the potential to be converted peripherally into estrogen $)^{23}$. 3) Previously hidden tumor-associated antigens, stress proteins and danger-associated molecular patterns will be exposed after radiotherapy ${ }^{24}$. The exposure of antigens might activate antitumor immunity ${ }^{25}$ and stimulate tumor cell killing.

In addition, we sought to identify those individuals who would benefit the most from radiotherapy. Lumpectomy combined with radiotherapy reduced the risk of ipsilateral second BC compared with lumpectomy alone. Among patients younger than 40 years, Black women with high-grade tumors were likely to benefit most. A possible reason could be that the second cancer risk is substantially greater among patients with initial diagnosis of breast cancer at the age of $<50$ years and high-grade tumors has a higher-grade malignancy and a greater accumulation of genetic mutations and epigenetic modifications which related to a second primary cancer and higher mortality. Therefore, these patients could benefit more due to their possible higher risk of mortality and MPC. Patients with a negative ER status benefited more in terms of mortality and MPC. Although data from endocrine therapy use are not included in the SEER database, it is likely that some ER-positive patients received hormone treatment combined with radiotherapy; thus, the mortality benefit from radiation appeared artificially low. In addition, the IDEAL trial (BOOG 2006-05) found that adjuvant endocrine therapy did not have a statistically significant effect on either overall survival or distant recurrence but reduced the risk of MPC $(\mathrm{HR}=0.39,95 \% \mathrm{CI}: 0.19-0.81, \log -\mathrm{rank} \mathrm{p}=0.01)^{26}$. Considering the benefit of reducing the risks of mortality and $\mathrm{MPC}$, we recommend that patients undergo radiotherapy whether receiving endocrine therapy or not.

Several studies have pointed out the relationship between recurrence and mortality and the benefit of radiation. In RTOG 9804, a good-risk subset of patients with mammographically detected low- or intermediate-grade DCIS, measuring less than 2.5 $\mathrm{cm}$ with margins $\geq 3 \mathrm{~mm}$, the ipsilateral local failure rate was decreased significantly with the addition of radiotherapy ${ }^{6}$. In 2018 , Vasily Giannakeas et al studied 140,366 patients with DCIS diagnosed between 1998 and 2014 in the SEER 18 database. The adjusted HR for mortality associated with radiotherapy (based on 29,465 propensity-matched pairs) was 0.77 (95\% CI: $0.67-0.88$; $\mathrm{P}<0.001)^{27}$. A recent population-based cohort study observed that the rate of ipsilateral invasive BC for women undergoing breast-conserving surgery with no record of radiotherapy was higher than that for women receiving radiotherapy (adjusted rate ratio $1.43,95 \%$ confidence interval 1.05 to 1.96), and the mortality from BC between the various treatment groups was similar ${ }^{21}$. In our study, we found that radiotherapy could reduce the risk of death, and the HR was 0.744 (95\%CI: $0.7139-0.7761)$ among the cohort. However, the reduction in death only occurred in the patients with only one primary cancer, and radiotherapy did not have a statically significant effect among the MPC patients. We further compared mortality and found that the occurrence of MPC led to a higher risk of death than no occurrence of MPC. Therefore, radiotherapy reduced the risk of MPC rather than the death associated with MPC and thereby reduced overall mortality.

\section{Limitations}

Our study has several limitations. First, this is a retrospective study from the SEER database rather than a prospective cohort study, so inherent selection biases may undermine its external validity. Second, similar to other SEER database studies, this study lacks specific information on chemotherapy and hormone treatment. However, our PSM model controlled age, year of diagnosis, pathologic grade, hormone receptor status and tumor size, which determined the patients' systemic therapy usage. On the other hand, comparison between SIRs of second primary cancer after DCIS for patients treated with lumpectomy or lumpectomy plus radiotherapy showed that only statistical significance observed in second female breast cancer, demonstrated a relatively small 
impact on non-breast tumor brought by chemotherapy and hormone treatment. Third, the treatments for patients were not assigned at random. In addition, cases with some unknown variables were excluded; thus, the results may not include all patients with DCIS.

\section{Conclusion}

Among patients with DCIS, treatment with lumpectomy plus radiotherapy was associated with a significant reduction in mortality and risk of MPC, mainly second primary BC. Younger patients, Black women with high-grade tumors were likely to benefit most. The effect of the sequence of radiation and surgery and the form of radiotherapy should be explored in future studies.

Supplementary Materials: provided in the supplementary file.

Author Contributions: Yitong Li and Wenjun Yi finished the methodology and data curation, Yitong Li prepared the original draft preparation and Qitong Chen, Dengjie Ouyang and Taohong reviewed and edited the manuscript. Wenjun Yi visualized and supervised the research. All authors have read and agreed to the published version of the manuscript.

Funding: This research received no external funding.

Conflicts of Interest: The authors declare no conflict of interest.

\section{References}

1

Cowell, C. F. et al. Progression from ductal carcinoma in situ to invasive breast cancer: revisited. $\mathrm{Mol}$ Oncol 7, 859-869, doi:10.1016/j.molonc.2013.07.005 (2013).

Veronesi, U. et al. Twenty-Year Follow-up of a Randomized Study Comparing Breast-Conserving Surgery with Radical Mastectomy for Early Breast Cancer. 347, 1227-1232, doi:10.1056/NEJMoa020989 (2002).

3 Litière, S. et al. Breast conserving therapy versus mastectomy for stage I\&\#x2013;Il breast cancer: 20 year follow-up of the EORTC 10801 phase 3 randomised trial. The Lancet Oncology 13, 412-419, doi:10.1016/S1470-2045(12)70042-6 (2012).

Kunkler, I. H., Williams, L. J., Jack, W. J. L., Cameron, D. A. \& Dixon, J. M. Breast-conserving surgery with or without irradiation in women aged 65 years or older with early breast cancer (PRIME II): a randomised controlled trial. The Lancet Oncology 16, 266-273, doi:https://doi.org/10.1016/S1470-2045(14)71221-5 (2015).

Morrow, M. et al. Standard for Breast Conservation Therapy in the Management of Invasive Breast Carcinoma. CA: $A$ Cancer Journal for Clinicians 52, 277-300, doi:10.3322/canjclin.52.5.277 (2002).

McCormick, B. et al. RTOG 9804: A Prospective Randomized Trial for Good-Risk Ductal Carcinoma In Situ Comparing Radiotherapy With Observation. Journal of Clinical Oncology 33, 709-715, doi:10.1200/jco.2014.57.9029 (2015).

van Maaren, M. C. et al. 10 year survival after breast-conserving surgery plus radiotherapy compared with mastectomy in early breast cancer in the Netherlands: a population-based study. The Lancet Oncology 17, 1158-1170, doi:10.1016/s1470-2045(16)30067-5 (2016).

Effects of radiotherapy and of differences in the extent of surgery for early breast cancer on local recurrence and 15-year survival: an overview of the randomised trials. The Lancet 366, 2087-2106, doi:10.1016/s0140-6736(05)67887-7 (2005). $\begin{array}{lllll}\text { tissues } & \text { or } & \text { Concer } & \text { 231-237, }\end{array}$ doi:10.1002/1097-0142(196103/04)14:2<231::aid-cncr2820140203>3.0.co;2-2 (1961).

Silverman, B. G., Lipshitz, I. \& Keinan-Boker, L. Second Primary Cancers After Primary Breast Cancer Diagnosis in Israeli Women, 1992 to 2006. J Glob Oncol 3, 135-142, doi:10.1200/jgo.2016.003699 (2017).

Yi, M. et al. Other primary malignancies in breast cancer patients treated with breast conserving surgery and radiation therapy. Ann Surg Oncol 20, 1514-1521, doi:10.1245/s10434-012-2774-8 (2013). 
Li, Z., Wang, K., Shi, Y., Zhang, X. \& Wen, J. Incidence of second primary malignancy after breast cancer and related risk factors-Is breast-conserving surgery safe? A nested case-control study. Int J Cancer 146, 352-362, doi:10.1002/ijc.32259 (2020).

Goldstraw, P. et al. The IASLC Lung Cancer Staging Project: Proposals for Revision of the TNM Stage Groupings in the Forthcoming (Eighth) Edition of the TNM Classification for Lung Cancer. J Thorac Oncol 11, 39-51, doi:10.1016/j.jtho.2015.09.009 (2016).

Austin, P. C. Optimal caliper widths for propensity-score matching when estimating differences in means and differences in proportions in observational studies. Pharmaceutical Statistics 10, 150-161, doi:10.1002/pst.433 (2011).

Austin, P. C. \& Stuart, E. A. Moving towards best practice when using inverse probability of treatment weighting (IPTW) using the propensity score to estimate causal treatment effects in observational studies. Statistics in medicine 34, 3661-3679, doi:10.1002/sim.6607 (2015).

An, J. H. et al. A Possible Association Between Thyroid Cancer and Breast Cancer. Thyroid 25, 1330-1338, doi:10.1089/thy.2014.0561 (2015).

Withrow, D. R., Morton, L. M., Curtis, R. E., Schonfeld, S. J. \& Berrington de Gonzalez, A. Radiotherapy for ductal carcinoma in situ and risk of second non-breast cancers. Breast Cancer Res Treat 166, 299-306, doi:10.1007/s10549-017-4410-6 (2017).

Innos, K. \& Horn-Ross, P. L. Risk of second primary breast cancers among women with ductal carcinoma in situ of the breast. Breast Cancer Research and Treatment 111, 531-540, doi:10.1007/s10549-007-9807-1 (2008).

Kushner, C. J., Hwang, W. T., Wang, S., Solin, L. J. \& Vapiwala, N. Long-term risk of second malignancies in women after breast conservation therapy for ductal carcinoma in situ or early-stage breast cancer. Breast Cancer Res Treat 170, 45-53, doi:10.1007/s10549-018-4729-7 (2018).

Shaitelman, S. F. et al. Rates of Second Malignancies After Definitive Local Treatment for Ductal Carcinoma In Situ of the Breast. International Journal of Radiation Oncology*Biology*Physics 81, 1244-1251, doi:10.1016/j.ijrobp.2010.07.2005 (2011).

Mannu, G. S. et al. Invasive breast cancer and breast cancer mortality after ductal carcinoma in situ in women attending for breast screening in England, 1988-2014: population based observational cohort study. BMJ 369, m1570, doi:10.1136/bmj.m1570 (2020).

Parveen, B. et al. Risk of Second Primary Thyroid Cancer after Radiotherapy for a Childhood Cancer in a Large Cohort Study: An Update from the Childhood Cancer Survivor Study. Radiation Research 174, 741-752, doi:10.1667/RR2240.1 (2010).

Inskip, P. D. Pelvic radiotherapy, sex hormones, and breast cancer. Cancer Causes Control 5, 471-478, doi:10.1007/bf01694761 (1994).

Vatner, R. E., Cooper, B. T., Vanpouille-Box, C., Demaria, S. \& Formenti, S. C. Combinations of immunotherapy and radiation in cancer therapy. Front Oncol 4, 325, doi:10.3389/fonc. 2014.00325 (2014).

Suzuki, R. et al. Efficacy of radiation boost after breast-conserving surgery for breast cancer with focally positive, tumor-exposed margins. J Radiat Res 61, 440-446, doi:10.1093/jrr/rraa005 (2020).

Blok, E. J. et al. Optimal Duration of Extended Adjuvant Endocrine Therapy for Early Breast Cancer; Results of the IDEAL Trial (BOOG 2006-05). JNCI: Journal of the National Cancer Institute 110, 40-48, doi:10.1093/jnci/djx134 (2018).

Giannakeas, V., Sopik, V. \& Narod, S. A. Association of Radiotherapy With Survival in Women Treated for Ductal Carcinoma In Situ With Lumpectomy or Mastectomy. JAMA Netw Open 1, e181100, doi:10.1001/jamanetworkopen.2018.1100 (2018). 


\section{Supplementary Files}

This is a list of supplementary files associated with this preprint. Click to download.

- SupplementaryMaterials.pdf 